\title{
Left ventricular synchronicity in acromegaly
}

\author{
Ludovica F. S. Grasso • Annamaria Colao
}

Received: 12 May 2013/Accepted: 14 June 2013/Published online: 27 June 2013

(C) Springer Science+Business Media New York 2013

GH hypersecretion has a direct detrimental effect on the heart: a specific cardiomyopathy is a well-known consequence of acromegaly. The most common features of the acromegalic cardiomyopathy include concentric biventricular hypertrophy, left ventricular systolic and diastolic dysfunction, rhythm disturbances, and an increased prevalence of valve abnormalities [1]. Cardiac hypertrophy is present in about $20 \%$ of young normotensive patients with short duration of the disease and in up to $90 \%$ of hypertensive patients with long-term duration of the disease [2]. This evidence lends support to the concept that cardiac hypertrophy is an early event strongly worsening with the disease duration, and aggravated by hypertension. These cardiac complications are a major determinant of the shortened life expectancy of acromegalic patients. Indeed, patients with acromegaly display about $30 \%$ enhanced mortality rate, in particular, cardiovascular disease represents the cause of death in $60 \%$ of cases [3]. Ageing and long duration of GH/IGF-I excess are main determinants of cardiac derangement and early diagnosis and effective treatment are essential to reserved the acromegalic cardiomyopathy [4]. In fact, younger patients respond better to treatment in terms of cardiac improvement than middleaged patients, provided that all had controlled GH and IGFI levels [5].

L. F. S. Grasso · A. Colao ( $₫)$

Sezione di Endocrinologia, Dipartimento di Medicina Clinica e Chirurgia, Università Federico II di Napoli, Naples, Italy

e-mail: colao@unina.it

L. F. S. Grasso

e-mail: ludovicagrasso@hotmail.com
Besides cardiac structure and function have been deeply investigated in acromegaly, left ventricular synchronicity has never been evaluated so far. In their study, Kırış et al. [6] describe left ventricular synchronicity in acromegaly.

Left ventricular dyssynchrony (LVD), as defined by the standard deviation of time to peak systolic velocity by tissue Doppler imaging, reflects both electrical activation and the result of heterogeneity of contractile properties in the wall [7]. LVD is a common phenomenon in patients with heart failure, reduced left ventricular ejection fraction and wide QRS complexes, and it is associated with impairment of left ventricular systolic and diastolic function and adverse clinical outcomes [7, 8]. However, nowadays LVD seems to be a marker of early left ventricular dysfunction even before of regional wall motion abnormality or overt systolic and diastolic abnormalities, and it seems to occur even when the left ventricular ejection fraction is relatively preserved and QRS duration is within the normal range [9]. Previous data suggest that factors other than ischemia might be involved, such as intramyocardial fibrosis or matrix changes that might affect electrical conduction [7].

Later, it was shown that left ventricular synchronicity might be impaired also in patients who have narrow QRS complexes in disorders such as hypertension, diabetes mellitus, and hypo/hyperthyroidism [6].

Moreover, few report recently demonstrated the relationship of left ventricular hypertrophy $(\mathrm{LVH})$ and LVD in hypertensive patients, suggesting that hypertension impairs left ventricular function not only by influencing myocardial function, but also by impairing left ventricular synchronicity [8]. In particular, Seo et al. [10] reported that systolic LVD during exercise is significantly associated with the degree of $\mathrm{LVH}$ in hypertensive patients, despite normal echocardiograms at rest, demonstrating that dynamic LVD using exercise echocardiography is probably one of the 
important topics in the hypertensive heart disease, because it will provide prognostic information.

Based on these findings, Kırış et al. [6] first studied the presence of LVD in patients with active acromegaly, a class of patients with similar ultrastructural myocardial and metabolic abnormalities, due to an increased incidence of arterial hypertension, $\mathrm{LVH}$, diabetes mellitus, and systolic and diastolic dysfunction during the final stage of the acromegalic cardiomyopathy. However, all the patients with the common alterations related with LVD, as well as diabetes mellitus, uncontrolled hypertension, atrial flutter or fibrillation, prolonged QRS duration ( $\geq 120 \mathrm{msn}$ ), reduced left ventricular ejection fraction (LVEF $<50 \%$ ), significant valvular heart disease, hypertrophic cardiomyopathy, chronic renal disease, heart failure, and coronary artery disease were excluded from the study, and it was demonstrated that left ventricular synchronicity was impaired in these class of acromegalic patients, suggesting a possible direct hormone effects on cardiac synchronicity, independently of age's patients and disease duration. Moreover, the baseline echocardiographic parameters such as the left ventricular ejection fraction and left ventricular diameters were similar in acromegalic patients and controls, whereas prevalence of LVH was higher in acromegaly group, and it was found a significant relationship between LVD and LVH in univariate analysis, suggesting that the myocardial hypertrophy might be responsible of LVD resulting in ineffective contraction.

Nevertheless, further investigation is needed to define the role of GH and IGF-I excess on the cardiac synchronicity, to investigate the effect of treatment of acromegaly on the LVD, and to clarify the necessity in the clinical practice, to perform in these patients an echocardiographic measurements extended to evaluate the cardiac synchronicity, especially in acromegalic patients with well-known cardiovascular complications. Moreover, based on previous articles, evaluation of the exerciseinduced dyssynchrony in these population of patients could be interesting, because it is likely that dyssynchrony might worsen or increase on exercise and contribute significantly to global ventricular dysfunction and symptoms of breathlessness.

\section{References}

1. A. Colao, D. Ferone, P. Marzullo, G. Lombardi, Systemic complications of acromegaly: epidemiology, pathogenesis, and management. Endocr. Rev. 25(1), 102-152 (2004). doi:10.12 10/er.2002-0022

2. A. Colao, R. Pivonello, L.F. Grasso, R.S. Auriemma, M. Galdiero, S. Savastano, G. Lombardi, Determinants of cardiac disease in newly diagnosed patients with acromegaly: results of a 10 year survey study. Eur. J. Endocrinol. 165(5), 713-721 (2011). doi: 10.1530/eje-11-0408

3. M. Arosio, G. Reimondo, E. Malchiodi, P. Berchialla, A. Borraccino, L. De Marinis, R. Pivonello, S. Grottoli, M. Losa, S. Cannavò, F. Minuto, M. Montini, M. Bondanelli, E. De Menis, C. Martini, G. Angeletti, A. Velardo, A. Peri, M. Faustini-Fustini, P. Tita, F. Pigliaru, G. Borretta, C. Scaroni, N. Bazzoni, A. Bianchi, M. Appetecchia, F. Cavagnini, G. Lombardi, E. Ghigo, P. BeckPeccoz, A. Colao, M. Terzolo, Italian Study Group of Acromegaly, Italian Study Group of Acromegaly. Predictors of morbidity and mortality in acromegaly: an Italian survey. Eur. J. Endocrinol. 167(2), 189-198 (2012). doi:10.1530/EJE-12-0084

4. A. Colao, The GH-IGF-I axis and the cardiovascular system: clinical implications. Clin. Endocrinol. (Oxf.) 69(3), 347-358 (2008). doi:10.1111/j.1365-2265.2008.03292.x

5. A. Colao, P. Marzullo, A. Cuocolo, L. Spinelli, R. Pivonello, D. Bonaduce, M. Salvatore, G. Lombardi, Reversal of acromegalic cardiomyopathy in young but not in middle-aged patients after 12 months of treatment with the depot long-acting somatostatin analogue octreotide. Clin Endocrinol (Oxf). 58(2), 169-176 (2003). doi:10.1046/j.1365-2265.2003.01689.x

6. Kırış A, Erem C, Turan OE, Civan N, Kırış G, Nuhoğlu I, Ilter A, Ersöz HO, Kutlu M. Left ventricular synchronicity is impaired in patients with active acromegaly. Endocrine. (2012). doi: 10.1007/s12020-012-9859-9

7. C.M. Yu, H. Lin, Q. Zhang, J.E. Sanderson, High prevalence of left ventricular systolic and diastolic asynchrony in patients with congestive heart failure and normal QRS duration. Heart 89, 54-60 (2003). doi:10.1136/heart.89.1.54

8. P. Lancellotti, M. Moonen, Left ventricular dyssynchrony: a dynamic condition. Heart Fail. Rev. 17(6), 747-753 (2012). doi: 10.1007/s10741-011-9275-x

9. M. Wang, G.H. Yan, W.S. Yue, C.W. Siu, K.H. Yiu, S.W. Lee, C.P. Lau, H.F. Tse, Left ventricular mechanical dyssynchrony impairs exercise capacity in patients with coronary artery disease with preserved left ventricular systolic function and a QRS duration $\leq 120$ ms. Circ. J. 76(3), 682-688 (2012). doi:10.1253/ circj.CJ-11-0735

10. H.S. Seo, Y.H. Cho, J.H. Choi, J. Suh, N.H. Lee, O.K. Lim, The association of left ventricular hypertrophy with intraventricular dyssynchrony at rest and during exercise in hypertensive patients. J Cardiovasc Ultrasound 20, 174-180 (2012). doi:10.4250/jcu. 2012.20.4.174 